\title{
Archéopages
}

Archéopages

Archéologie et société

41 | 10/2014-01/2015

La place du cheval

\section{Chronostratigraphie et paléoenvironnements des fonds de vallée du bassin français de l'Escaut}

Laurent Deschodt

\section{(2) OpenEdition}

1 Journals

Édition électronique

URL : https://journals.openedition.org/archeopages/1077

DOI : 10.4000/archeopages. 1077

ISSN : 2269-9872

Éditeur

INRAP - Institut national de recherches archéologiques préventives

Édition imprimée

Date de publication : 1 août 2015

Pagination : 98-99

ISSN : 1622-8545

Référence électronique

Laurent Deschodt, « Chronostratigraphie et paléoenvironnements des fonds de vallée du bassin français de l'Escaut », Archéopages [En ligne], 41 | 10/2014-01/2015, mis en ligne le 01 janvier 2017 , consulté le 23 janvier 2022. URL : http://journals.openedition.org/archeopages/1077 ; DOI : https:// doi.org/10.4000/archeopages. 1077

Ce document a été généré automatiquement le 23 janvier 2022.

(c) Inrap 


\title{
Chronostratigraphie et paléoenvironnements des fonds de vallée du bassin français de l'Escaut
}

\author{
Laurent Deschodt
}

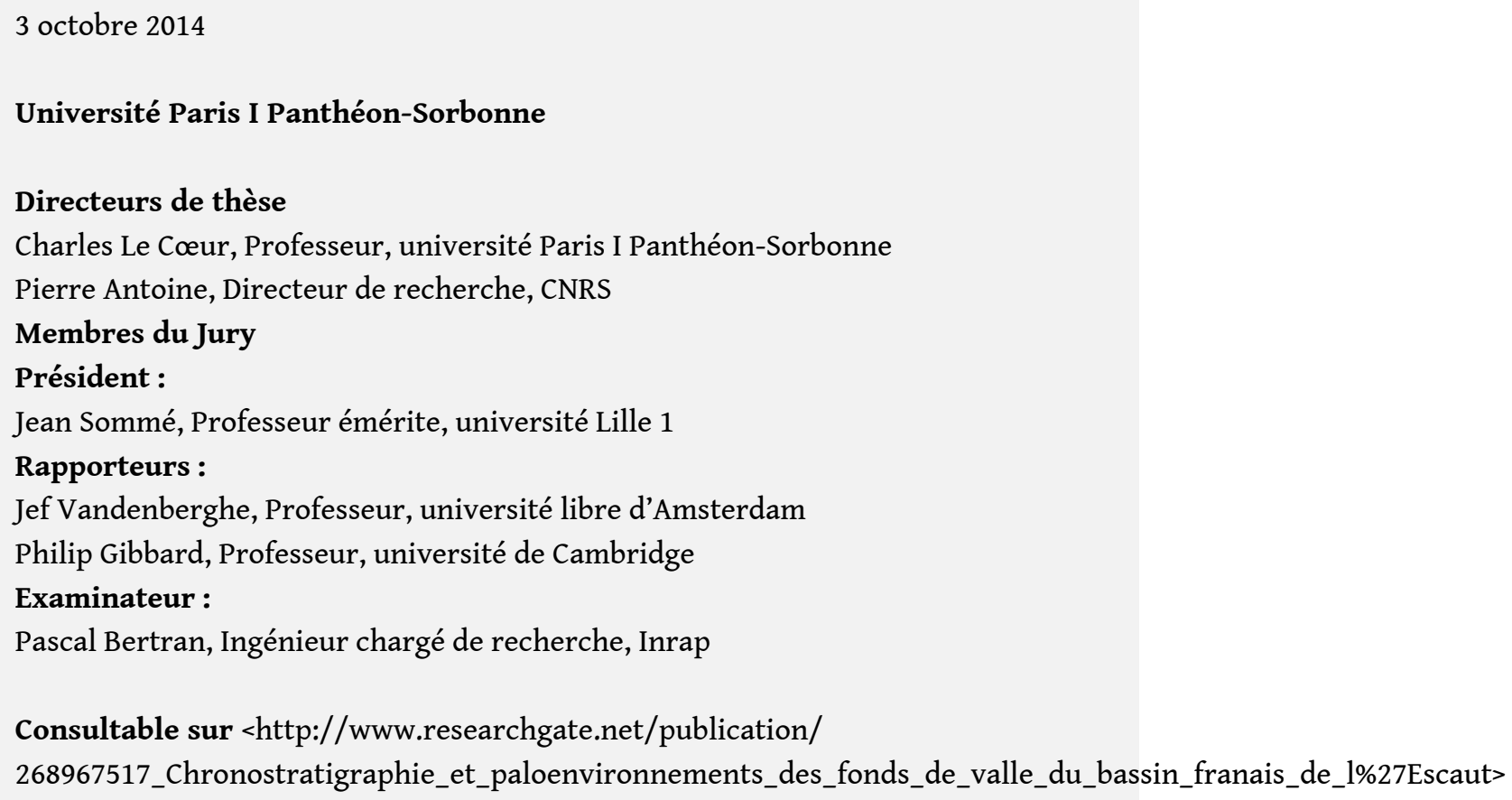

1 Le bassin français amont de l'Escaut est composé d'un haut pays (plateaux crayeux) et d'un bas pays (plaines basses et collines de sables et d'argiles). Il est caractérisé par l'absence de cours d'eau d'importance et, dans le bas pays, par une couverture sédimentaire quaternaire continue et des pentes faibles. Le comblement quaternaire des fonds de vallée est relativement peu étudié malgré l'intérêt certain des 
enregistrements. Ceux-ci peuvent être classés en trois groupes: les dépôts du Pléniglaciaire weichselien et les dépôts antérieurs ou postérieurs.

Parmi les dépôts antérieurs au Pléniglaciaire, nous distinguons ceux du Début Glaciaire weichselien des dépôts plus anciens. Le Début Glaciaire peut être conservé sur de grandes surfaces dans le bas pays à la faveur d'une protection par les loess postérieurs et la progradation des versants (exemple de la vallée de la Deûle dans la région de Lille). Le pédostratotype du Début Glaciaire de Warneton (plaine de la Lys) est commenté. Nous émettons un doute quant à son attribution chronologique. À notre avis, une nouvelle étude serait nécessaire pour le confirmer ou éventuellement le rajeunir. Dans tous les cas, il s'agit d'une formation tourbeuse weichselienne d'intérêt majeur qui mérite des études environnementales complémentaires. Les dépôts de fonds de vallée antérieurs au Weichselien sont rencontrés sous trois formes: (a) des nappes graveleuses accumulées en piedmont du haut pays pendant plusieurs cycles climatiques (sud de la plaine de la Lys, plaine de confluence Escaut-Haine), (b) des dépôts eemiens isolés et à faible profondeur, conservés à la faveur des aléas de l'évolution paléogéographique des vastes plaines (site de Waziers dans la plaine de la Scarpe), (c) des dépôts fluviatiles du Saalien et de l'Eemien à une vingtaine de mètres de profondeur dans la plaine de la Lys et dans la partie aval de la Deûle et de la Marque. Il s'agit alors du comblement de la "vallée flamande ", en continuité avec la partie aval belge. La chronostratigraphie et les variations des pentes longitudinales du bed rock dans la partie française suggèrent une morphogenèse récente (depuis le Saalien) probablement liée à l'évolution paléogéographique du bassin de la mer du Nord. Le remblaiement fluvio-éolien weichselien qui scelle les dépôts antérieurs apparaît comme atypique à l'échelle du Pléistocène et pourrait également être lié aux mêmes évolutions paléogéographiques. Dans tous les cas, les enregistrements sédimentaires du Pléistocène moyen suggèrent un bilan tectonique proche de la stabilité à l'échelle du Quaternaire. Ceci est en conformité avec la position géographique de la zone, entre un Bassin parisien en surrection et un bassin belgo-néerlandais en subsidence. Le fait participe à la mauvaise conservation des dépôts les plus anciens. Une attention particulière a été portée aux enregistrements pléniglaciaires d'activités fluviatiles sur versant et dans les extrémités amont du réseau de talwegs. Quelques indices suggèrent que le développement vers l'amont du réseau de talwegs est dû à de courtes phases morphogénétiques en lien avec des phénomènes thermokarstiques. Les périodes du Pléniglaciaire inférieur et moyen weichseliens sont mal documentées. Des indices indirects suggèrent une forte activité fluviatile pendant le Pléniglaciaire inférieur. Quelques formations limoneuses enfouies dans les plaines sont attribuées, sans certitude, au Pléniglaciaire moyen. La base du Pléniglaciaire supérieur est érosive. La période se divise en: (a) une phase d'activité fluviatile intense et étendue jusque dans les extrémités amont des réseaux et sur les versants (contexte de pergélisol), (b) à partir d'environ $22 \mathrm{ka}$, une forte rétractation du réseau hydrographique actif et un remblaiement fluvio-éolien massif, ce dernier en continuité stratigraphique avec la couverture loessique sur versant. Une déflation permet localement la croissance de dunes (en particulier dans la plaine de la Scarpe). Un modèle d'évolution de petite vallée en zone loessique à la fin du Pléniglaciaire supérieur weichselien est proposé. L'héritage morpho-sédimentaire de la fin du Pléniglaciaire weichselien est prépondérant. Il justifie la subdivision stratigraphique proposée. 
3 Le Tardiglaciaire est principalement connu à travers les enregistrements complémentaires d'Houplin-Ancoisne et de Dourges. Ils sont proposés comme stratotypes pour le bas pays et comparés à une quinzaine d'autres sites dans la zone d'étude. La présence de zones lacustres apparente le bas pays du nord de la France aux plaines plus septentrionales. Des divergences constatées dans l'évolution des niveaux d'eau à la fin du Bølling et au début de l'Allerød pourraient être un indice de variation des répartitions saisonnières des précipitations. Les données disponibles sur le Dryas récent autorisent la subdivision suivante : (a) un début sec vers 11,0 ka BP, comparable à l'Intra Allerød Cold Period, (b) le développement d'un pergélisol discontinu (Dourges étant l'évidence la plus méridionale), (c) une crise érosive sur versant (développement rapide de ravines), (d) une activité fluviatile régulière entre environ 10,7 et 10,4 ka $\mathrm{BP}$, (e) une phase particulièrement sèche.

4 La fragmentation des données sur l'Holocène limite les possibilités de synthèse. Nous proposons toutefois quelques thèmes à considérer comme problématiques de recherches : (a) bipartition de l'Holocène, avec d'abord une tendance à l'extension de tourbe ou de travertin jusqu'à une phase d'incision (fin Atlantique ou début Subboréal), vraisemblablement sous commande principale climatique (mais sans synchronisme strict à l'échelle d'une région ni même d'un cours d'eau) ; (b) différentes périodes de fortes activités hydrologiques, voire de crises érosives locales : fin Atlantique/début Subboréal ?, vers 3,0 ka BP ?, vers le II siècle de notre ère ?; (c) dépôt massif de limon ou d'argile d'inondation lié aux activités médiévales.

Une synthèse est proposée pour chaque sous-bassin versant, accompagnée de réflexions méthodologiques, de prospectives et de propositions de recherches. Toutes les propositions ne peuvent être exécutées dans le strict cadre de notre activité en archéologie préventive (notamment l'étude du comblement profond de la vallée flamande). Toutefois, l'archéologie préventive peut indéniablement être une pourvoyeuse majeure de connaissances dès que les observations stratigraphiques sont complétées par quelques analyses.

\section{AUTEUR}

\section{LAURENT DESCHODT}

Inrap, UMR 8591 « Laboratoire de géographie physique : environnements quaternaires et actuels " 